\title{
Effectiveness of alkali and sodium bicarbonate treatments on sugar palm fiber: mechanical, thermal, and chemical investigations
}

\begin{abstract}
Sugar palm fiber (SPF) as one of the attractive natural fibers to reinforce matrix is gaining attention. This is largely due to its similar properties when compared with other established natural fibers. The aim of this study is to investigate the effectiveness of sodium bicarbonate as a treatment chemical for SPF in comparison with established alkaline treatment. Both treated and untreated fibers were characterized and it was found that the treated fiber shows an increase in crystallinity, thermal stability, and surface's roughness when compared with the untreated. Among the two different treatments, SPF treated with alkali has an initial decomposition temperature of $255.47^{\circ} \mathrm{C}$, while sodium bicarbonate treated and untreated fibers have $250.19^{\circ} \mathrm{C}$ and $246.76^{\circ} \mathrm{C}$, respectively. In both cases, the thermal stability of the fiber was improved. Also, as revealed by the X-ray diffraction (XRD) analysis, the crystallinity index of SPF treated with alkali and sodium bicarbonate increased by $18.43 \%$ and $13.60 \%$, respectively, when compared with untreated fiber. In conclusion, the investigation proved that treatment with the sodium bicarbonate has a significant effect on the physicochemical properties of SPF and the chemical could be an alternative chemical for treating other cellulose fibers.
\end{abstract}

Keyword: Alkali; Chemical composition; Chemical treatment; Sodium bicarbonate; Sugar palm fiber; Thermal property 